\title{
§ 41 Strafvorschriften
}

Mit Freiheitsstrafe bis zu einem Jahr oder mit Geldstrafe wird bestraft, wer

1. entgegen $\S 4$ Abs. 2 Satz 1 in Verbindung mit Satz 2 ein Medizinprodukt in den Verkehr bringt,

2. entgegen $\S 6$ Abs. 1 Satz 1 ein Medizinprodukt, das nicht den Vorschriften der Strahlenschutzverordnung oder der Röntgenverordnung unterliegt oder bei dessen Herstellung ionisierende Strahlen nicht verwendet wurden, in den Verkehr bringt oder in Betrieb nimmt,

3. entgegen $\S 6$ Abs. 2 Satz 1 in Verbindung mit einer Rechtsverordnung nach $\$ 37$ Abs. 1 ein Medizinprodukt, das nicht den Vorschriften der Strahlenschutzverordnung oder der Röntgenverordnung unterliegt oder bei dessen Herstellung ionisierende Strahlen nicht verwendet wurden, mit der CE-Kennzeichnung versieht,

4. entgegen $\S 20$ Absatz 1 Satz 1 oder Satz 4 Nummer 1 bis 6 oder Nummer 9 , jeweils auch in Verbindung mit $\$ 20$ Absatz 4 oder Absatz 5 oder $\$ 21$ Nummer 1 oder entgegen § 22b Absatz 4 mit einer klinischen Prüfung beginnt, eine klinische Prüfung durchführt oder eine klinische Prüfung fortsetzt,

5. entgegen $\S 24$ Satz 1 in Verbindung mit $§ 20$ Absatz 1 Satz 1 oder Satz 4 Nummer 1 bis 6 oder Nummer 9, jeweils auch in Verbindung mit $\$ 20$ Absatz 4 oder Absatz 5, oder entgegen $\S 24$ Satz 1 in Verbindung mit $\S 22 b$ Absatz 4 mit einer Leistungsbewertungsprüfung beginnt, eine Leistungsbewertungsprüfung durchführt oder eine Leistungsbewertungsprüfung fortsetzt oder

6. einer Rechtsverordnung nach $\S 37$ Abs. 2 Satz 2 zuwiderhandelt, soweit sie für einen bestimmten Tatbestand auf diese Strafvorschrift verweist.

Kommentierung siehe $\S 43$ 